\title{
Philosophiques
}

\section{L'histoire du thomisme québécois ou de l'importance de mettre les âmes à leur place}

\section{Pierre Thibault}

Volume 8, numéro 2, octobre 1981

URI : https://id.erudit.org/iderudit/203173ar

DOI : https://doi.org/10.7202/203173ar

Aller au sommaire du numéro

Éditeur(s)

Société de philosophie du Québec

ISSN

0316-2923 (imprimé)

1492-1391 (numérique)

Découvrir la revue

Citer cet article

Thibault, P. (1981). L'histoire du thomisme québécois ou de l'importance de mettre les âmes à leur place. Philosophiques, 8(2), 343-348.

https://doi.org/10.7202/203173ar d'utilisation que vous pouvez consulter en ligne.

https://apropos.erudit.org/fr/usagers/politique-dutilisation/ 


\title{
ÉTUDE CRITIQUE
}

\section{L'HISTOIRE DU THOMISME QUÉBÉCOIS OU \\ DE L'IMPORTANCE DE ME'TTRE LES ÂMES À LEUR PLACE*}

\author{
par Pierre Thibault
}

Soutenue à l'Université Laval en janvier 1978, cette thèse était vivement attendue. Sa publication s'imposait. Les travaux de Marc Lebel sur L'enseignement de la philosophie au Petit Séminaire de Québec (1765-1880) (Thèse de maîtrise, Université Laval, 1964) et ceux ' l'auteur lui-même sur L'enseignement de la philosophie au College de Montréal (1790-1876) (Thèse de maîtrise, Université Laval, 1969) avaient éloquement montré l'intérêt de ce genre de recherche et l'intérêt d'un travail analogue portant sur l'ensemble du Québec. L'auteur lui-même avait ici et là annoncé, dans diverses publications, qu'il s'était engagé dans ce travail, et la qualité de ce que nous avions déjà de lui nous rendait confiants qu'il allait s'en acquitter honorablement.

Nous ne sommes pas déçus. L'ampleur et l'aridité de la documentation originale qu'il a localisée et dépouillée constituait un défi énorme. Comme on pouvait le prévoir, l'auteur a bien relevé ce défi, avec ce qu'il fallait, à la fois, de discernement et d'acharnement.

L'ampleur même, mais aussi la diversité, la difficulté d'accès et le caractère rébarbatif de cette documentation rendaient d'avance toute tentative d'analyse aléatoire et périlleuse. L'auteur n'en a pas fait une analyse approfondie. Il est évident qu'il n'en avait pas la prétention. Il n'en est pas moins parvenu à en faire une

* Lamonde, Yvan. La philosophie et son enseignement au Québec (1665-1920), Montréal, Hurtubise HMH, 1980. Les propos qui suivent s'inspirent d'une communication présentée, dans le cadre de l'ACFAS, à la Société de philosophie du Québec, à Sherbrooke, le 3 mai 1981. 
description cohérente et pertinente à un niveau bien circonscrit, ni sociologique, évitant de s'aventurer dans une analyse politique globale, ni structurelle, faisant l'économie d'une analyse interne systématique.

Ces limites de son travail, sur lesquelles je reviens plus bas, relèvent en principe d'une bonne méthodologie et ne sauraient lui être reprochées. Maintenant que la documentation a été rendue accessible et que nous en avons une analyse descriptive à un niveau assez significatif pour servir de fil d'Ariane à ceux qui viennent après lui, l'auteur lui-même, et d'autres que lui, seront en mesure d'aller puiser plus commodément les matériaux requis pour la confection d'autres chapitres.

\section{L'INTERROGATOIRE DES SOURCES.}

Je prends un premier exemple: le texte qui commence à la page 91 et qui montre les positions politiques affichées dans les «Cours» autour de la polémique mennaisienne, avant et après la condamnation de 1834 des Paroles d'un croyant, et débouchant sur les Institutiones de Demers. La démonstration est ici essentiellement empirique: elle se base sur les propos proprement politiques que contiennent des éthiques dont l'ampleur croît rapidement. Dans la mesure où les sources elles-mêmes ont un caractère éclectique, cette méthode éclectique d'analyse leur convient sans doute. Elle comporte l'inconvénient de ne pas mettre en évidence le caractère proprement conjoncturel de cette approche éclectique, le caractère lui-même éclectique des velléités thomistes qui vont suivre, et à sous-estimer ultérieurement le caractère spécifiquement anti-éclectique du thomisme romain. Au début, le thomisme n'est pas préconisé en raison de ses positions politiques (celles-ci constituent même un handicap à première vue), mais en raison de son ontologie dont on sait, sans toujours le dire, qu'elle est le plus solide fondement d'une certaine politique.

À cet égard, ces pages font bien sentir la panique postmennaisienne qui appelle le thomisme. Le mennaisisme, fraîchement anathématisé pour des raisons politiques si l'on veut, mais des raisons politiques motivées par la structure même du système et non pas seulement par ses positions spécifiquement politiques, ce mennaisisme avait eu le temps de discréditer le cartésianisme, 
qui constituait jusqu'alors une alternative philosophicothéologique viable, et non seulement ne d'identifiait pas aux Lumières, mais offrait aux théologiens un terrain où affronter les Lumières. Le cartésianisme une fois discrédité, on cherchera désespérément un «rationalisme» (puisqu'on ne peut plus être traditionaliste) théologiquement idoine.

Cette limite de l'analyse est encore plus sensible dans la partie du chapitre III qui suit la page 154. L'auteur, ici, n'est plus embarrassé par des sources manuscrites éparses: il travaille sur un nombre fini de manuels imprimés.

Pourtant, l'ultramontanisme y est présenté comme synonyme univoque de l'union du trône et de l'autel, alors qu'il en a été autrement depuis les débuts, des querelles médiévales du Sacerdoce et de l'Empire jusqu'à l'époque récente de l'absolutisme royal, dont personne ne croit encore, dans ces milieux, qu'elle 'oit véritablement achevée. Que l'on puisse sporadiquement les identifier entre 1815 et 1860 est purement accidentel et relève de la tactique.

De même, la renaissance du droit naturel, signalée au passage, n'est pas un épiphénomène. Elle a une importance centrale et structurelle dans la restauration du thomisme. Les inconvénients de ce traitement linéaire apparaissent encore, par exemple, à partir de la page 161. Il ne s'agit plus seulement et surtout de rejeter la thèse du contrat social et la légitimité du régime républicain: il s'agit de récupérer de l'autre main ce mouvement pour damer le pion, après deux siècles où Rome avait rongé son frein, aux prétentions des princes catholiques. C'est seulement à ce niveau qu'on peut comprendre l'équivoque de l'identification conjoncturelle du traditionalisme et du thomisme, alors que le second se savait l'antithèse du premier.

\section{QUELLE HIÉRARCHIE?}

Ce qui caractérise la montée du thomisme, ce n'est pas l'apparition d'une hiérarchie ou l'accentuation de la structure hiérarchique de l'univers comme de la société; ou entre le pouvoir spirituel et le pouvoir temporel. Il n'y aurait rien là de bien neuf: tout au plus la réassertion moins timide de ce qui allait toujours de soi. 
Sous ces apparences obvies se joue une opération proprement subversive, qui concerne l'ontologie même, et donc la substance des relations hiérarchiques.

À une biérarchie des substances, qui évoquait une relative consubstantialité, une participation à des degrés divers à une substantialité homogène, donc à des prérogatives analogues (les différents degrés du spirituel) se substitue, comme au $13^{\mathrm{e}}$ siècle, une biérarchie des fins, entre des substances que sépare une distance infinie.

Et ce qui unit l'homme à Dieu n'est pas la spiritualité partagée, mais un lien gratuit, pur don d'un Dieu hors d'atteinte, lien dit surnaturel.

Ce qui importe, dans le choix des ontologies hiérarchisées, c'est l'endroit où elles situent la faille principale.

Ontologie platonico-augustinienne

Ontologie aritotélico-thomiste Dieu Incréé

Esprit

Anges

Ames

Matières

$$
\text { Corps }
$$

Créé

Autres

D'un côté, anges et «âmes» sont proches du divin, degrés inférieurs d'une même étoffe. De l'autre, ils en sont infiniment éloignés, dans le même camp que les corps.

\section{ALERTE À PARER OU OCCASION À SAISIR?}

On entrevoir que les querelles sur la nature des anges n'étaient pas purement spéculatives, et que les égards du «surnaturalisme» thomiste pour «l'autonomie de l'ordre naturel» n'étaient pas désintéressés.

À la faveur de l'alerte générale déclenchée par le rationalisme des Lumières et le républicanisme des Révolutions, l'Église romaine coupe littéralement l'herbe ontologique sous les pieds des théologiens et des princes prétendant partager un pouvoir «spirituel». 
Ce qui compte, en thomisme, c'est la légitimité surnaturelle et personne ne peut y prétendre - le mot le dit - en vertu de ce qu'il est.

La défense contre l'ennemi extérieur commun est l'occasion pour Rome de reprendre le terrain perdu dans une lutte interne millénaire. Dès le $\mathrm{II}^{\mathrm{e}}$ siècle, Tertullien s'affligeait

. . que Platon soit devenu, en toute bonne foi, le pourvoyeur de tous les hérétiques. C'est de lui, en effet, que vient cette thèse, enseignée dans le Phédon, que les âmes venues ici-bas proviennent de là-haut, et que d'ici elles retourneront là-haut . . .

L'année même de l'encyclique Aeterni Patris (1879) le philosophe Paul Janet observait finement que

. . .l'excès du spiritualisme ne plaît nullement à la théologie; c'est là même une des difficultés que Descartes eut à vaincre; il parut faire la part trop grande à l'indépendance naturelle de l'âme et à sa suprématie spirituelle. Le platonisme n'a pas toujours été bien vu dans l'Église; et c'est la philosophie d'Aristote qui a été généralement préférée. Les jésuites et l'école de Bonald se sont toujours opposés à la théorie des idées innées, théorie caractéristique de toute école idéaliste et spiritualiste. Tout ce qui affranchit l'âme relève trop l'orgueil humain'.

\section{LES LIMITES DU CONTEXTE LOCAL.}

Il est difficile de se garder d'une erreur de perspective qui vient de ce que l'on accorde trop d'importance au contexte local. La société canadienne à la fin du XIX ${ }^{\mathrm{e}}$ siècle, «société confessionnalisée et cléricalisée», n'est guère typique. La situation locale explique l'évolution institutionnelle de l'enseignement de la philosophie, et le monopole que l'Église exerce sur lui, ainsi que l'empressement spectaculaire de l'Église canadienne à adhérer au thomisme romain. Elle n'éclaire pas la genèse de la structure du discours philosophique, qui s'enracine en terrain plus large, mais en anime certains chapitres ou certains épisodes.

Une doctrine qui accolait «le droit naturel du peuple et le droit surnaturel du pape» (Feugueray), et qui «allait permettre au Pontife de contrôler les princes par le peuple» (Gilson) trouvait ici une situation à sa mesure, qui donnait le sentiment d'une harmo-

1. Paul Janet, La pbilosophie française contemporaine, Paris, Calmann Lévy, 1879, p. 13. 
nie pré-établie. Elle armait l'Église pour traiter avec un pouvoir hérétique issu de la conquête, comme elle lui permettait ailleurs de traiter avec les pouvoirs républicains issus des révolutions.

L'Église canadienne a joué un rôle essentiel dans ce que nous appelions naguère notre "survivance». Ce rôle comportait un délicat jeu de balance: assurer la soumission au pouvoir établi, bien qu'il fût étranger et hérétique, en exigeant de lui en retour qu'il tolère la survie de «la langue, gardienne de la foi». Gardienne, surtout, du monopole culturel sur les masses qui permettait justement aux clercs de tenir le pouvoir civil en respect.

La puissance de l'Église canadienne tenait à un subtil dosage dans la poursuite de ces deux objectifs, à première vue contradictoires. On comprend qu'elle ait embrassé éperdument le thomisme pontifical. Il avait pour vocation séculaire, non seulement de les concilier, mais de les conjuger ${ }^{2}$.

Yvan Lamonde nous fait cadeau d'un instrument de travail irremplaçable, qui va féconder la réflexion et la recherche de nombre d'entre nous. Y compris d'abord la sienne dont nous attendons encore beaucoup.

Il aura plaisir à relire maintenant à loisir des textes extrêmement riches qu'il a lui-même publiés dans son Historiograpbie de la philosopbie au Québec (1853-1971), notamment ceux de Paquet, Robert, Bastien et Beauregard. Il y verra que les ténors de la «ligne romaine» étaient plus lucides qu'on pourrait le croire.

\section{Université Laval}

2. Pierre Thibault, Savoir et pouvoir: philosopbie thomiste et politique cléricale au $X I X^{\mathrm{e}}$ siècle. Québec, Presses Universitaires Laval, 1972, Avant-propos, p. XXVIII. 